\section{The effects of speed and accuracy strategies in an information-reduction task}

\author{
JAMES H. COOTS and WILLIAM A. JOHNSTON \\ University of Utah, Salt Lake City, Utah 84112
}

\begin{abstract}
Using an information-reduction task, choice reaction time (CRT) to digits presented visually was examined as a function of size of the positive set (memory load), set for speed vs accuracy, and presence vs absence of a backward mask. The mask was designed to delimit preprocessing time-the time available for readout from iconic storage. The intercept and slope of the function relating CRT to memory load were lower for speed set than accuracy set, but were largely unaffected by the mask. Since controlled reduction in iconic readout time neither affected the intercept directly nor altered the effect of set on the intercept, the effects of set cannot be attributed to the initial stage of information processing. The data take issue with prior interpretations of the effect of set in terms of stimulus preprocessing, and document the important contribution of output processing to CRT and variations therein.
\end{abstract}

In a visual choice reaction time (CRT) task, $S$ is shown one of a number of alternative stimuli to which he attempts to make the appropriate one of a number of alternative responses. Choice reaction time is a measure of the elapsed time between stimulus onset and the response. An information-reduction task involves the special case in which the number of altemative stimuli exceeds the number of alternative responses. In its most common form, this task contains only two responses, positive and negative, and two or more atimuli may form the basis for each response. The stimuli to which the positive response is appropriate comprise the positive set, and those to which the negative response is appropriate comprise the negative set.

Smith (1968) has outlined four stages of information processing which contribute to CRT. In Stage 1, or stimulus preprocessing, the stimulus information enter a temporary store referred to by Neiner (1967) as the icon, and a stimulus model or representation is formed from that information. The duration of Stage 1 is limited, at least in part, by the rate of decay of the icon. In Btage 2, or stimulus categorization, the stimulus model produced by Stage 1 is compared somehow with the memory model comprising the positive set in an effort to secure a match. The duration of stage 2 is limited by the central comparison rate, or the rate at which the stimulus model is compared with the memory models of the positive set. Somewhere along the line, time must be devoted to the retrieval of the positive set from memory so that the memory models will be available for comparison with the stimulus model. However, it is not clear to which stage this retrieval should be attributed; it may occur during either Stages 1 or 2, or perhaps even as a preparatory operation prior to stimulus onset. In Stage 3, or response selection, a response is chosen and programmed on the basis of the outcome of Stage 2; and in Stage 4, or response execution, that response is actually produced. If the outcome of Stage 2 is a match, then the positive response should be selected and executed; otherwise, the negative response should be made.

A determination of the processing stages to which the effect of important variables on CRT may be localized would greatly enhance our understanding of human information processing. Sternberg (1966) has advanced a technique for accomplishing this task which involves first reducing the empirical relationship between CRT and size of the positive set-or memory load (ML)-to a linear equation, and then identifying the two parameters of that equation with different procesaing stages. Memory load may or may not have to be transformed to yield a linear CRT function. Thus, the banic equation is CRT = A + B (ML), where $A$ is ascumed to represent Stages 1,3 , and 4 (total encoding and decoding) and $B$ is taken to represent Stage 2 (central comparison).

One variable which has received a good deal of attention in human information procensing is that of set for speed is accuracy. In an extension of Sternbers's basic procedure, Briger has endeavored to identify the proceuning stages to which the effect of this variable on CRT may be attributed (Briggs \& Swanson, 1970; Swans on \& Brigg, 1969). The conclusion that Briggs derived from his data was that the effects of set are limited to Stage 1. Specifically, accuracy set produced a higher intercept (A) than speed set, but the slopes (B) were equal, indicating that the effect was realized somewhere in Stages 1, 3, and 4. It was reasoned that Stages 3 and 4 were unlikely loci, since the latter is defined as purely motor and the former involved only a binary decision-positive or negative. Thus, Stage 1 was pinpointed as the locus of the effect of set by elimination. It was postulated that $S$ simply reads out less information from the icon under a speed set than under an accuracy set. This finding was obtained both when the stimuli were unfamiliar random forms (Swanson \& Briggs, 1969) and when they were familiar letters (Briggs \& Swanson, 1970). Briggs's emphasis on Stage 1 , and the rationale by which he dismissed Stages 3 and 4 from further consideration, is entirely in keeping with the recent trend of researchers in this area to emphasize input over output processing in explanations of overt behavior. This trend appears to be somewhat precarious, considering how little has been determined empirically about the output stages ( 3 and 4 ) of human information processing.

The present study attempted to test Briggs's conclusion by experimentally manipulating the duration of Stage 1. Logically, if Stage 1 is the true locus of the effect of set and if the duration of Stage 1 can be controlled experimentally, such control should enable one to attenuate, or even eliminate, the effect of set on CRT. Specifically, set differences should be reduced when $\mathrm{Ss}$ in the accuracy condition are not allowed to indulge in Stage 1 processing in excess of that performed by $S e$ in the speed condition. Control of the readout from iconic storage, and thus of Stage 1, was attempted by uning a backward masking procedure.

\section{METHOD}

Bubjects and Danign

Eight male univernity student recruited through the atudent employment office cerved individually for seven 75-min sevions. All si participated on Thureday and Friday of one week, and Monday through Friday of the next week. The fint 8 days were devoted to practice, and the last four to the experiment proper. In addition to a base wage of $\$ 2.00$ per rearion. Ss received incentive pay according to a system described below.

The baric derion was a 2 by 2 by 3 factorial with repented measures on all factors: two levels of set (speed ve accuracy), two levels of mask (mask ve no mask control), and three levels of ML $(1,2$, and 4). Whereas $\mathrm{ML}$ was manipulated within each session, both set and mask were manipulated between sessions. The four combinations of set and mask will be 


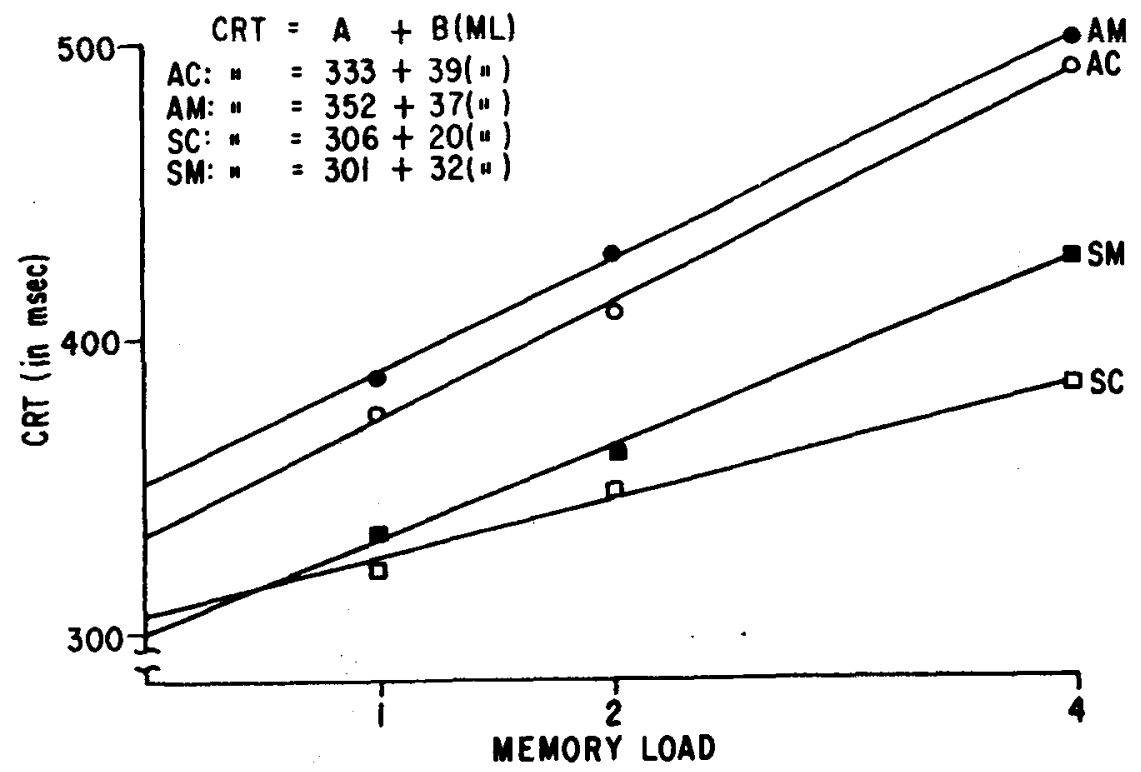

Fig. 1. Mean of median CRT as a function of memory load for each combination of set and mask.

referred to as accuracy control (AC), accuracy mask (AM), speed control (SC), and speed mask (SM). Response mode (positive vs negative) was also a variable, but was not considered since it had virtually no effect on CRT.

\section{Task and Apparatus}

The S's task was to decide whether a digit presented tachistoscopically was a member of a positive or a negative set, indicate his decision by pushing the appropriate response button as quickly as possible, and then report his confidence in the accuracy of the response. Confidence ratings varied from 1 to 5 as follows: $1(5)=$ absolutely sure the response was incorrect (correct); $2(4)=$ think the response was incorrect (correct), but not sure; $3=$ uncertain whether the response was correct or incorrect.

Black cutouts of the 10 digits $0-9$ were mounted on white cards and presented in a Scientific Prototype two-channel tachistoscope. The height of each stimulus subtended a visual angle of approximately $.87 \mathrm{deg}$. The stimulus presentation rate was S-paced, one stimulus occurring about every 9-15 sec. Whenever $S$ was ready, he said "GO!," and E pushed the stimulus exposure button as quickly as possible. A millisecond timer which started at stimulus onset measured the CRT for both the positive (left index finger) and negative (right index finger) responses. The $E$, separated from $S$ by an opaque screen, recorded S's CRT, confidence rating (CR), and response accuracy (indicated by response lights on a control panel). The $E$ provided $S$ with speed (fast or slow) and accuracy (correct or incorrect) feedback after each response. It took approximately $7 \mathrm{sec}$ for $E$ to record the data from the previous trial, provide feedback, reset the apparatus, and inform $S$ that the next trial could begin. Throughout the task, $\mathbf{S}$ wore a headset through which $E$ delivered instructions and feedback between trials and presented a 65-dB white noise to mask extraneous sounds during trials.

\section{Procedure}

In every session (except the third) $S$ responded to three series of 60 digits, each series consisting of four contiguous blocks of 15 digits and restricted to a single level of ML $(1,2$, or 4 digits). All three levels of $\mathrm{ML}$ (60-digit series) were presented in each session. Following Stermberg (1966, Experiment 2), each block of 15 digits was constructed to keep both stimulus and response entropies constant; regardless of $M L$, the relative frequency of positive responses in each block was $4 / 15$. Within this system, the assignment of specific digits was determined randomly. The constitution of the positive set was announced approximately $2 \mathrm{~min}$ before the start of the digit series for each level of $\mathrm{ML}$, and $\mathrm{S}$ was asked to repeat the positive set at various points throughout the digit series. There was a 5-min break between series.

In each practice session exposed for $1 \mathrm{sec}$ and were never followed by a visual mask, and all Ss received the same order of ML levels across digit series. Set instructions on the practice sessions directed Ss to emphasize speed and accuracy equally (Sessions 1-3), the stimuli were and were augmented by a monetary incentive system in Sessions 2 and 3 . The Ss were told that they could earn a maximum of $\$ 3.00$ incentive pay in each of the last two practice sessions and that $50 \%$ of this pay would be based on each criterion (speed and accuracy). During Session 1, the speed feedback given by $\mathrm{E}$ was the actual CRT in milliseconds. During Sessions 2 and 3 , however, the criterion for speed feedback (fast or slow) was the mean for the previous session under the same level of ML.

The third practice session involved only 15 digit presentations for each ML condition. The remainder of the session was devoted to a psychophysical test to determine the exposure duration required for $\mathrm{S}$ to correctly identify digits $90 \%$ of the time when stimulus exposure was terminated by a visual noise mask. The masking stimulus was simply a $3 \times$ 3 in. array of randomly positioned pieces of black material against a white background. The luminance was $3.2 \mathrm{~mL}$ for both stimulus and mask fields. Using the method of constant stimuli, the obtained exposure durations ranged across $\mathrm{S} s$ from 14 to $63 \mathrm{msec}$, with a mean of $31 \mathrm{msec}$. These exposure durations were used throughout the remainder of the experiment. The results of this procedure defined for each $\mathbf{S}$ the two levels of mask to which he was exposed on the experimental seasions (Sessions 4-7). Stimulus exposure was followed by the mask in Conditions $A M$ and $S M$ and by a dark field in Conditions $A C$ and SC.

It was assumed that effective readout of iconic information was limited to stimulus exposure duration under mask conditions, but allowed to run its full course under control conditions. Since it could be angued that Sis were able tolextract decaying stimulus information from the icon even after mask onset (Eriksen \& Eriksen, 1971), an empirical test was made of the extent to which $\mathrm{Ss}$ could read through the mask when stimulus and mask were exposed simultaneously. ${ }^{1}$ This test was conducted on an independent but comparable group of eight Ss. The upshot of this test was that stimulus identification performance was essentially chance level (10\% correct) when stimulus-mask onset asynchrony was zero. This held true when stimulus exposure duration was that determined for the $90 \%$ threshold ( $8.5 \%$ correct), and even when it was $350 \mathrm{msec}(16.5 \%)$, a liberal upper bound of the icon duration (Eriksen \& Eriksen, 1971). Hence, it appears safe to conclude that the mask did severely degrade, if not terminate, iconic readout of stimulus information. 
The presentation order of ML levels on each experimental session was partially counterbalanced by giving half the Ss one order of ML levels and the other half a different order. The specific orders used varied between sessions. As on the practice sessions, set was manipulated via instructions and a monetary incentive system. In each experimental session, however, $90 \%$ of the $\$ 3.00$ maximum incentive pay was based upon the emphasized criterion (speed or accuracy). For each level of $\mathrm{ML}$, the feedback criterion for speed throughout the experimental sessions was S's mean CRT for the final practice series under that level of ML. The intersession order in which Ss received Conditions $\mathbf{A C}, \mathbf{A M}, \mathbf{S C}$, and SM adhered to a counterbalancing scheme.

\section{RESULTS AND DISCUSSION}

Each $S$ yielded 60 CRTs (four blocks of trials) at each combination of set, mask, and ML. However, in order to focus analysis on those trial blocks in which $\mathbf{S}$ was most attentive, the block in which he performed most poorly in terms of correct and fast responces was eliminated. Moreover, of the remaining 45 trials per level of $M L$, only those on which $\mathbf{S}$ responded correctly were considered for CRT analysis. For each $\mathrm{S}$, the median CRT of correct responses for each level of ML was computed, and these data are summarized in Fig. 1. The distinction between positive and negative responses was disregarded in the computation of medians, since a preliminary analysis had indicated that CRTs from the two response modes were virtually equal.

All four curves in Fig. 1 describe CRT as a linear function of ML. An analycis of variance applied to the data revealed that all three main effects were simifieant: set, $F(1,7)=26.13$, $\mathrm{p}<.01$; malk, $\mathrm{F}(1,7)=22.63$, $p<.01$; and $M L, F(2,14)=62.42$, p $<.001$. Thus, overall CRT was greater under accuracy set than under speed set, greater under mask than under control, and a direct function of ML. The only interaction attaining significance was Set by ML $F(2,14)=$ $5.20, p<.05$. The basis of this interaction was that $M L$ produced a greater effect on CRT under accuracy set than under speed set, indicating that set affected the slope as well as the intercept of the CRT function. The data were further examined by computing the $A$ and $B$ parameters of the CRT equation for each $S$ under each combination of set and mask. Analyses of variance disclosed a main effect of set on both $A[F(1,7)=6.01$, $p<.05]$ and $B[F(1,7)=19.32$, $p<.01]$. None of the remaining sources of variance approached significance for either A or B (p > .10).

The results of all the analyses clearly reveal that both the intercept and the slope of the CRT function were lower under speed set than under accuracy set. The intercept difference confirms the Swaneon and Briggs (1969) data, but the slope difference conflicts with their data. In the present study, the speed set apparently accelerated both Stage 2 (B) and at least one of the remaining stages (A). Although not confirmed by the analyses of $A$ and $B$, the visual mask tended to produce an overall increase in CRT. Certainly, the mask did not reduce the intercept under either set, a fact squarely at odds with the Swanson and Briggs (1969) explanation of the effect of set on the intercept. Specifically, if the speed set reduced the intercept by limiting Stage 1 processing as Swanson and Briggs hypothesized, then the intercept should also be reduced when Stage 1 processing is limited experimentally by a visual mak. The fact that the mask in the present work neither reduced the intercept nor in any way altered the effect of set on the intercept render the Swanion and Bring theais debatable.

These arguments are bolstered by

Table 1

Mean Number of Errors and Mean Confldance Rating (CR) as a Function of Set, Mast, and Memory Load

\begin{tabular}{|c|c|c|c|c|c|}
\hline \multirow[b]{2}{*}{ Mearure } & \multirow[b]{2}{*}{ Mats } & \multirow[b]{2}{*}{ Set } & \multicolumn{3}{|c|}{ Memory Lond } \\
\hline & & & 1 & 2 & 4 \\
\hline \multirow[b]{2}{*}{ Error* } & Control & $\begin{array}{l}\text { Aceurncy } \\
\text { Speed }\end{array}$ & 2.25 & $\begin{array}{r}.38 \\
8.38\end{array}$ & $\begin{array}{r}.60 \\
8.88\end{array}$ \\
\hline & Mak & $\begin{array}{l}\text { Accuracy } \\
\text { Speed }\end{array}$ & $\begin{array}{r}.60 \\
2.88\end{array}$ & $\begin{array}{l}1.00 \\
3.63\end{array}$ & $\begin{array}{l}2.00 \\
5.38\end{array}$ \\
\hline \multirow[b]{2}{*}{$\mathbf{C R}$} & Control & $\begin{array}{l}\text { Accuracy } \\
\text { Speed }\end{array}$ & $\begin{array}{l}4.98 \\
4.78\end{array}$ & $\begin{array}{l}4.96 \\
4.73\end{array}$ & $\begin{array}{l}4.98 \\
4.67\end{array}$ \\
\hline & Mark & $\begin{array}{l}\text { Accuracy } \\
\text { Speed }\end{array}$ & $\begin{array}{l}4.96 \\
4.74\end{array}$ & $\begin{array}{l}4.81 \\
4.67\end{array}$ & $\begin{array}{l}4.80 \\
4.48\end{array}$ \\
\hline
\end{tabular}

*Each mean error could assume a maximum value of 45.00 .
Table 2

Type of Error as a Function of Set and Mask

\begin{tabular}{|c|c|c|c|}
\hline \multirow[b]{2}{*}{ Mask } & \multirow[b]{2}{*}{ Set } & \multicolumn{2}{|c|}{ Type of Error* } \\
\hline & & $\begin{array}{l}\text { Preper- } \\
\text { ceptual }\end{array}$ & $\begin{array}{l}\text { Postper- } \\
\text { ceptual }\end{array}$ \\
\hline Control & $\begin{array}{l}\text { Accuracy } \\
\text { Speed }\end{array}$ & $\begin{array}{l}\mathbf{1} \\
\mathbf{2}\end{array}$ & $\begin{array}{r}7 \\
65\end{array}$ \\
\hline Mask & $\begin{array}{l}\text { Accuracy } \\
\text { Speed }\end{array}$ & $\begin{array}{l}11 \\
13\end{array}$ & $\begin{array}{l}10 \\
66\end{array}$ \\
\hline
\end{tabular}

*Preperceptual and postperceptual errors were defined operationally as errors followed by high (4 or 5) and low (I or 2) confidence ratings, respectively.

the response accuracy and $C R$ data of the present experiment, which are summarized in Table 1 . Whereas only set $[F(1,7)=32.79, p<.001]$ and $M L$ $[F(2,14)=18.63, p<.001]$ attained significance in an analysis of variance of total errors, all three main effects and the Mask by $\mathrm{ML}$ interaction achieved significance in the case of mean CR: $F(1,7)=41.39, p<.001$ for set, $F(1,7)=6.77, p<.05$ for mask, $F(2,14)=14.00, p<.001$ for $M L$, and $F(2,14)=4.55, p<.05$ for Mask by ML. Both response accuracy and $C R$ were reduced by the speed set and were an inverse function of $\mathrm{ML}$. In addition, CR was reduced by the visual mask, an effect which increased with ML.

Of more concern to the issues in question were the CRs which followed response errors. Errors can be classified as preperceptual and postperceptual: the former is based on an erroneous perception, and the latter is based on a correct perception followed by erroneous response selection or response execution. When an error was followed by a CR of 4 or 5 , it may be inferred that 8 was sure he had reaponded correctly, even though he had, in fact, erred, and the error may be classified as preperceptual. On the other hand, when an error was followed by a CR of 1 or 2 , it may be inferred that 8 knew he had erred, and the error mas be clanified $*$ postperceptual. The procensing stage affected by a variable should chow up in the type of error produced. If a variable primarily affects Btave 1, then it should affect the number of preperceptual errom more than the number of pontperceptual error; just the revere should be true if the variable primarily affects Stares 3 or 4 . Table 2 depicts the total number of errors of each type, pooled across Ss, for Conditions AC, AM, SC, and SM. Analyais via a dependent-measures $t$ test revealed that set had a marked effect on the frequency of postperceptual errors, $t(7)=9.92, p<.01$, and no effect on the frequency of preperceptual errors. This fact offers independent support 
to the conclusion gleaned from the CRT data that the effect of set is not attributable to Stage 1. Furthermore, mask did affect preperceptual errors primarily, and therefore did affect Stage 1, as was intended, $t(7)=1.97$ $\mathrm{p}<.05$, by a one-tailed test.

In brief, since the effect of set on the intercept was not altered when Stage 1 was severely curtailed by a visual mask, that effect calls for an interpretation that does not appeal to Stage 1. To which of the remaining three stages of information processing can the effect of set on CRT be attributed? The fact that set affected the slope of the CRT function in the present study suggests that it can influence Stage 2; however, this influence must be considered elusive, if not spurious, since it did not emerge in Briggs's research (Briggs \& Swanson, 1970; Swanson \& Briggs, 1969). Stages 3 and 4 seem to be the only available candidates for the locus of the reliable effect of set on the intercept. Because of its rather innocuous character, Stage 4 seems an unlikely prospect. This leaves Stage 3 as the only remaining alternative. One possibility is that $\mathbf{S}$ carries out a double-check operation under accuracy set that he omits under speed set. That is, $\mathbf{S}$ may recheck the outcome of Stage 2 before selecting a response under accuracy set; this rechecking time should be constant across levels of ML and could therefore account for the intercept difference between accuracy and speed sets.

A final comment is in order concerning the apparent tendency for researchers to overestimate drastically the contribution of Stage 1 to the intercept of the CRT function. Since the mask limited effective readout in Stage 1 to an average maximum of only 31 msec in the present study and yet did not reduce the intercept, it may be inferred that the average duration of Stage 1 under control conditions was not substantially greater than 31 msec. It follows that Stage 1 accounts for about $10 \%$ of the mean intercept value of $325 \mathrm{msec}$. This estimate of Stage 1 duration is substantially lower than that arrived at by Briggs and Swanson (1970) in a comparable study. ${ }^{2}$

In conclusion, various findings of the present research support a common theme: output processing (Stages 3 and 4) accounts for a sizeable portion of CRT and variations therein. Previous investigators have attributed substantially more to Stage 1 than seems justified on the basis of the present findings. This overemphasis on Stage 1, accompanied by a tendency to dismiss output processing, must be rectified if CRT research is to make a viable contribution to the understanding of human information processing.
BRIGGS, G. REFERENCES Encoding, decoding, and central functions in human information processing. Journal of Experimental Psy chology, 1970, 86, 296-308.

ERIKSEN, C. W., \& ERIKSEN, B. A. Visual perceptual processing rates and backward and forward masking. Journal of Experimental Psychology, 1971, 89, 306-313.

NEISSER, U. Cognitive psychology. New York: Appleton-Century-Crofts, 1967. Pp. 15-45.

SMITH, E. E. Choice reaction time: An analysis of the major theoretical positions. Psychological Bulletin, 1968, 69, 77-110.

STERNBERG, S. High-speed scanning in human memory. Science, 1966, 153, 652-654.

SWANSON, J. M., \& BRIGGS, G. E. Information processing as a function of speed versus accuracy. Journal of Experimental Psychology, 1969, B1, 223-229.

\section{NOTES}

1. This test was suggested by Charles $W$. Eriksen

2. Briggs and Swanson (1970) estimated the iconic readout rate to be about $150 \mathrm{msec}$ per bit of information transmitted, and identical analyses applied to the present data yielded an estimate of $83 \mathrm{msec}$ per bit. Both estimates are far in excess of that suggested by the controlled Stage 1 durations of the present study. Hence, the analytical technique by which Briggs and Swanson derived their estimate must be questioned.

(Received for publication February 16 , 1972.) 